\title{
Desmoid Tumor Mimicking Port Site Recurrence after Laparoscopic Retroperitoneal Lymph Node Dissection for Metastatic Testicular Tumor: A Case Report
}

\author{
Yujiro Hayashi ${ }^{*}$, Yu Ishizuya ${ }^{1}$, Ken Takeda1, Yuichiro Yamaguchi ${ }^{1}$, Masashi Nakayama ${ }^{1}$, \\ Yasuyuki Arai', Ken-Ichi Kakimoto', Yasuhiko Tomita'2, Kazuo Nishimura1 \\ ${ }^{1}$ Department of Urology, Osaka Medical Center for Cancer and Cardiovascular Diseases, Osaka, Japan \\ ${ }^{2}$ Department of Pathology, Osaka Medical Center for Cancer and Cardiovascular Diseases, Osaka, Japan \\ Email: yujiro8840103@gmail.com, isizuya-yu@mc.pref.osaka.jp, takeda-ke@mc.pref.osaka.jp, \\ yamaguchi-yu@mc.pref.osaka.jp, nakayama-ma@mc.pref.osaka.jp, arai-ya@mc.pref.osaka.jp, \\ kakimoto-ke@mc.pref.osaka.jp, tomita-ya@mc.pref.osaka.jp, nisimura-ka2@mc.pref.osaka.jp
}

Received 16 April 2015; accepted 18 May 2015; published 20 May 2015

Copyright @ 2015 by authors and Scientific Research Publishing Inc.

This work is licensed under the Creative Commons Attribution International License (CC BY).

http://creativecommons.org/licenses/by/4.0/

c) (7) Open Access

\begin{abstract}
Background: Desmoid tumor is a rare benign soft tissue tumor which commonly affects the trunk and extremities. Case presentation: We report a patient who presented with a tumor that slowly grew at the left lower abdominal port site 1 year after post-chemotherapy laparoscopic retroperitoneal lymph node dissection (RPLND) for metastatic testicular tumor. The enlarging mass mimicking port site recurrence after laparoscopic retroperitoneal lymph node dissection for metastatic testicular tumor was diagnosed as a desmoid tumor. Conclusion: To our knowledge, this is the first report of a desmoid tumor that developed after laparoscopic RPLND for a testicular tumor.
\end{abstract}

\section{Keywords}

Testicular Tumor, Retroperitoneal Lymph Node Dissection, Desmoid Tumor, CTNNB1

\footnotetext{
${ }^{*}$ Corresponding author.
}

How to cite this paper: Hayashi, Y., et al. (2015) Desmoid Tumor Mimicking Port Site Recurrence after Laparoscopic Retroperitoneal Lymph Node Dissection for Metastatic Testicular Tumor: A Case Report. Case Reports in Clinical Medicine, 4, 179-183. http://dx.doi.org/10.4236/crcm.2015.45036 


\section{Introduction}

Desmoid tumor is a rare benign soft tissue tumor with no characteristic imaging findings. This disease is diagnosed by not only imaging studies like CT scan or MRI but histological examinations such as biopsy It often occurs in the trunk and extremities, and surgical resection is indicated if the tumor grows. The recurrence rate is high at $19 \%-39.3 \%$ [1]-[3], and follow-up is often selected as the first choice [4]. We report a patient with a desmoid tumor which had gradually grown in the left lower abdominal port site 1 year after post-chemotherapy laparoscopic RPLND for metastatic testicular tumor; the tumor is diagnosed as desmoid tumor.

\section{Case Presentation}

A 24-year-old man visited our hospital with a chief complaint of painless enlargement of the scrotum. He was diagnosed as a left testicular tumor with retroperitoneal lymph node metastasis (non-seminoma, pT2N1M0S1). After 3 cycles of BEP therapy (bleomycin, etoposide, and cisplatin) according to the IGCCCG risk classification, he underwent laparoscopic RPLND (retroperitoneal approach). The dissected area was the left template, which was described by Weissbach and Boedefeld [5]. After removing the tumors, the left spermatic cord was also dissected to the internal inguinal ring. Pathological assessment of surgical specimens revealed no evidence of remaining viable tumor or teratoma. One year after the surgery, the patient felt a mass under the scar of the port site in the left abdomen used for RPLND, and abdominal computed tomography showed a $2.1 \mathrm{~cm}$ extraperitoneal mass. Because tumor markers such as alpha-fetoprotein, human chorionic gonadotrophin (hCG), and $\beta$ hCG were negative, the patient opted for follow-up. However, the tumor gradually enlarged, reaching $4.3 \times 3.2$ $\mathrm{cm}^{2}$ years after RPLND (Figure 1). Given that the tumor was near the port site scar, port site recurrence was strongly suspected. Thus tumorectomy was decided. The mass was about the size of a small chicken egg. On palpation, the mass was elastic hard with poor mobility. Apararectal incision was made under spinal anesthesia, and the mass was removed. The cut surface was homogeneous and white (Figure 2). Histological examination showed spindle-shaped cell proliferation and abundant collagen fibers, and immunohistological staining results were desmin (-), cytokeratin-MNF116 (-), and $\beta$-catenin (+) (Figure 3). Accordingly, a desmoid tumor was diagnosed. Gene mutation examination, using a paraffin-embedded block of the resected specimen, revealed S45P mutation in CTNNB1 encoding $\beta$-catenin.

\section{Discussion}

Desmoid tumor is a rare disease that develops in 2 to 4 of every million people; it appears more commonly in people in their 10 s to 40 s with a male to female ratio of 1 to 2, commonly affecting the trunk and extremities. Female hormones and trauma including surgical scars are reportedly associated with occurrence of desmoid tumors [1]. Although surgical resection is indicated if the tumor enlarges, the recurrence rate is high at $19 \%-39.3 \%$ [1]-[3], and follow-up is often adopted as the treatment of first choice [4]. Desmoid tumors have no characteristic imaging features, whereas spindle-shaped cell proliferation and abundant collagen fibers are revealed on his tological examination. Demoid tumor is diagnosed when intranuclear accumulation of $\beta$-catenin is observed.

Mutations of CTNNB1 and APC genes related to the Wnt pathway, which can regulate cell growth, are involved in the occurrence of desmoid tumors; in children, desmoid tumors can be the initial manifestation of familial adenomatous polyposis. Mutation in exon 3 of CTNNB1 (gene encoding $\beta$-catenin) results in increased translocation of $\beta$-catenin to the nucleus, thereby activating cell growth. CTNNB1 mutation is observed in approximately $90 \%$ of patients with idiopathic desmoid tumors, and mainly 3 types of mutations (T41A, S45F, and S45P) are reported [6] [7]. Although differentiation from low-grade sarcoma, fibroblastic lesions, and myofibroblastic lesions is important in diagnosing desmoid tumors, in some cases it is difficult to diagnose these tumors based on biopsy tissues. Confirmation of CTNNB1 mutation reportedly contributes to a definitive diagnosis [7]; we extracted DNA from a paraffin-embedded block of the resected tumor and confirmed exon 3 (S45P) mutation in CTNNB1 by PCR (Figure 4).

Desmoid tumor following testicular tumor treatment is rare. A total of 5 patients, including our patient, have been reported (4 patients received chemotherapy and underwent RPLND, and 1 received radiotherapy). The median period from completion of treatment to onset of desmoid tumor was 2 years (range, 1 to 19 years).

All were suspected to have testicular tumor recurrence and underwent surgical resection [8]-[11]. 


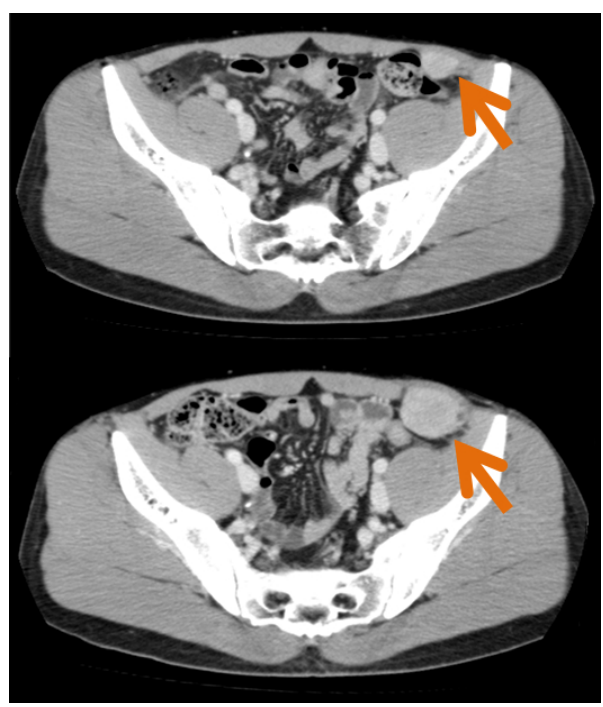

Figure 1. Abdominal CT scans. The growing tumor of left lower abdomen, one year (top) and two years (bottom) after laparoscopic retroperitoneal lymph node dissection, respectively.

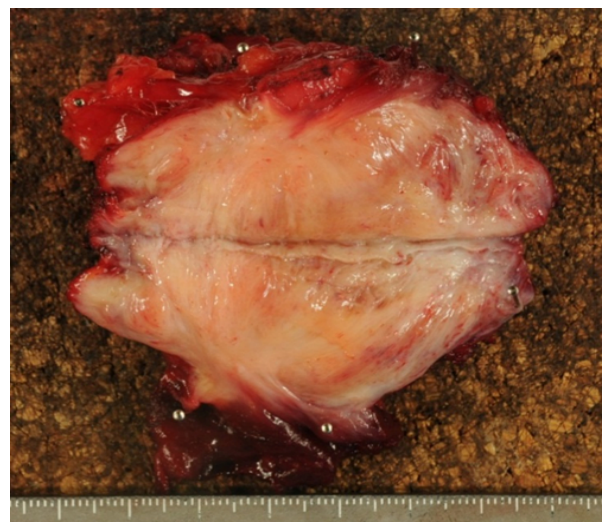

Figure 2. A cut surface of the resected tumor.

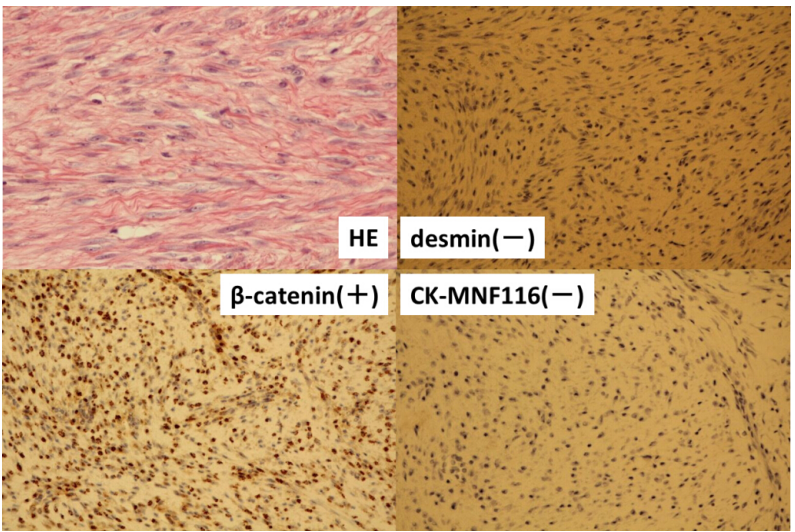

Figure 3. HE and immunohistochemical stains of the tumor. Spindle-shaped cell proliferation and abundant collagen fibers and $\beta$-catenin accumulation in nucleus. 


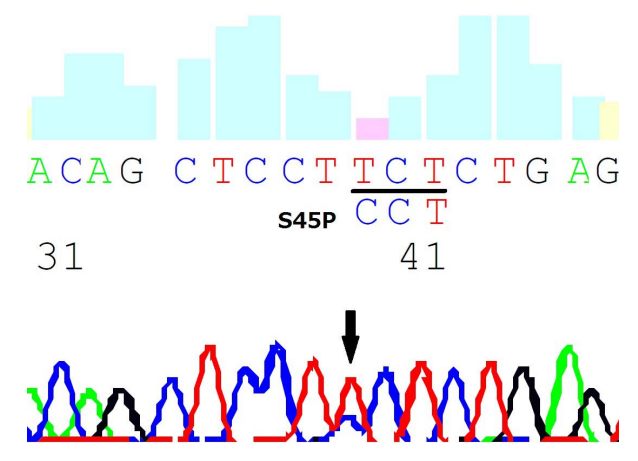

Figure 4. Mutation in CTNNB1. TCT-CCT mutation at nucleotide 133 (Ser-Pro at codon 45).

\section{Conclusion}

Desmoid tumor may occur at surgical sites, it is important to differentiate desmoid tumor from tumor recurrence and teratoma. We identified CTNNB1 mutation (S45P) for definite diagnosis. To our knowledge, this is the first report of a desmoid tumor that developed at the port site of laparoscopic RPLND.

\section{Competing Interests}

There are non-financial competing interests.

\section{References}

[1] Shields, C.J., Winter, D.C., Kirwan, W.O. and Redmond, H.P. (2001) Desmoid Tumours. European Journal of Surgical Oncology, 27, 701-706. http://dx.doi.org/10.1053/ejso.2001.1169

[2] Lazar, A.J., Tuvin, D., Hajibashi, S., Habeeb, S., Bolshakov, S., Mayordomo-Aranda, E., et al. (2008) Specific Mutations in the $\beta$-Catenin Gene (CTNNB1) Correlate with Local Recurrence in Sporadic Desmoid Tumors. The American Journal of Pathology, 173, 1518-1527. http://dx.doi.org/10.2353/ajpath.2008.080475

[3] Wang, Y.-F., Guo, W., Sun, K.-K., Yang, R.-L., Tang, X.-D., Ji, T. and Tang, S. (2015) Postoperative Recurrence of Desmoid Tumors: Clinical and Pathological Perspectives. World Journal of Surgical Oncology, 13, 26. http://dx.doi.org/10.1186/s12957-015-0450-8

[4] Salas, S., Dufresne, A., Bui, B., Blay, J.-Y., Terrier, P., Ranchere-Vince, D., et al. (2011) Prognostic Factors Influencing Progression-Free Survival Determined from a Series of Sporadic Desmoid Tumors: A Wait-and-See Policy According to Tumor Presentation. Journal of Clinical Oncology, 29, 3553-3558. http://dx.doi.org/10.1200/JCO.2010.33.5489

[5] Weissbach, L. and Boedefeld, E.A. (1987) Localization of Solitary and Multiple Metastases in Stage II Nonseminomatous Testis Tumor as Basis for a Modified Staging Lymph Node Dissection in Stage I. The Journal of Urology, 138, 77-82.

[6] Wang, W.L., Nero, C., Pappo, A., Lev, D., Lazar, A.J. and López-Terrada, D. (2012) CTNNB1 Genotyping and APC Screening in Pediatric Desmoid Tumors: A Proposed Algorithm. PediatrDevPathol, 15, 361-367. http://dx.doi.org/10.2350/11-07-1064-OA.1

[7] Le Guellec, S., Soubeyran, I., Rochaix, P., Filleron, T., Neuville, A., Hostein, I. and Coindre, J.-M. (2012) CTNNB1 Mutation Analysis Is a Useful Tool for the Diagnosis of Desmoid Tumors: A Study of 260 Desmoid Tumors and 191 Potential Morphologic Mimics. Mod Pathol, 25, 1551-1558. http://dx.doi.org/10.1038/modpathol.2012.115

[8] Lawatsch, E.J., Datta, M.W., Van Tuinen, P., Sudakoff, G.S., Davis, N.B. and Langenstroer, P. (2006) Intra-Abdominal Desmoid Tumor Following Retroperitoneal Lymph Node Dissection for Testicular Germ Cell Tumor. International Journal of Urology, 13, 84-86. http://dx.doi.org/10.1111/j.1442-2042.2006.01231.x

[9] Gianis, T.J., Carey, P.M. and Bracken, R.B. (1987) Giant Desmoid Tumor of the Abdominal Wall Masquerading as Recurrent Testicular Cancer. The Journal of Urology, 138, 152-153.

[10] Little Jr, J.S. and Foster, R.S. (1992) Intra-Abdominal Desmoid Tumor: An Unusual Case of Recurrent Tumor in a Testis Cancer Patient. The Journal of Urology, 147, 1619-1621.

[11] Wegner, H.E., Fleige, B. and Dieckmann, K.P. (1994) Mesenteric Desmoid Tumor 19 Years after Radiation Therapy for Testicular Seminoma. Urologia Internationalis, 53, 48-49. http://dx.doi.org/10.1159/000282632 


\section{List of Abbreviations}

RPLND: retroperitoneal lymph node dissection. 